\title{
Uma abordagem por videoanálise da propagação de um pulso em uma catenária
}

Video analysis approach concerning pulse propagation on a catenary

\author{
V. L. B. de Jesus ${ }^{* 1}$, D. G. G. Sasaki² \\ ${ }^{1}$ Instituto Federal de Educação, Ciência e Tecnologia do Rio de Janeiro, Campus Nilópolis, Nilópolis, RJ, Brasil \\ ${ }^{2}$ Centro Federal de Educação Tecnológica Celso Suckow da Fonseca, Unidade Maracanã, Rio de Janeiro, RJ, Brasil
}

Recebido em 1 de dezembro de 2015. Revisado em 17 de fevereiro de 2016. Aceito em 19 de março de 2016.

Em geral, os artigos na área de ensino sobre propagação de pulsos ou de ondas estacionárias utilizam fios ou arames muito leves, cujos pesos são desprezíveis diante das tensões aplicadas. Essa aproximação simplifica tanto a equação de onda, tornando-a linear, como a relação entre a tensão e a velocidade da onda. Entretanto, esse procedimento restringe os experimentos e exige o uso de sensores e/ou equipamentos mais caros para extrair dados confiáveis. Diferentemente, o objetivo deste trabalho foi criar e analisar um experimento didático barato e eficiente para a obtenção da relação entre a velocidade e a tensão de um pulso e da densidade linear de uma corda, cuja massa não fosse desprezível. Nessa situação, a corda não fica em linha reta, mas pende devido ao seu próprio peso formando uma curva chamada catenária. Para a coleta de dados, foram empregados somente uma câmera de filmagem de um smartphone e um software gratuito de videoanálise. Os diferentes modelos teóricos aplicados na interpretação dos resultados permitiram uma compreensão mais ampla e profunda do experimento. A análise dos resultados revelou uma variação da acurácia e precisão de acordo com os modelos matemáticos adotados. Isso possibilitou uma interessante visualização de que o trabalho experimental não depende somente dos equipamentos empregados, mas é indissociavelmente entrelaçado com as respectivas teorias. Além disso, as diferentes formas de modelar um mesmo fenômeno, com uma gradação de complexidade, proporcionam abordagens ajustadas para os níveis médio e superior, bem como para os diversos cursos de graduação.

Palavras-chave: ensino de física, videoanálise, velocidade da onda, catenária.

In general, the articles in the teaching area on pulses propagation or standing waves use very light wires or whose weights are negligible compared to the applied tensions. This approach both simplifies the wave equation making it linear as the relationship between the tension and the speed of the wave. However, this procedure restricts the experiments and requires the use of sensors and/or more expensive equipments to extract reliable data. In contrast, the aim of this work was to create and analyze a cheap and efficient educational experiment to obtain the relationship between the speed and the tension of a pulse and the linear density of a rope, whose mass was not negligible. In this situation, the rope is not straight, but hangs due to its own weight forming a curve called catenary. For data collection were employed only a camera of a smartphone and a free video analysis software. Different theoretical models applied in interpreting the results enabled a wide and deeper understanding of the experiment. The results showed a variation in the accuracy and precision according to the mathematical models adopted. This provided an interesting view, that is, the experimental work depends not only on the equipment used, but is entangled with the respective theories. Also, the different ways of modeling the same phenomenon, with a gradation of complexity, provide approaches adjusted to the high school and superior levels as well as for the various undergraduate courses.

Keywords: physics teaching, video analysis, pulse velocity, catenary.

*Endereço de correspondência: vitor.jesus@ifrj.edu.br 


\section{Introdução}

Recentemente foram publicados alguns artigos na área de ensino sobre ondas em uma corda. O mais antigo usa sensores magnéticos acoplados a um computador com um programa simulando um osciloscópio. Seus objetivos são mostrar a relação entre tensão e velocidade de propagação de um pulso em um arame metálico tensionado por massas calibradas, bem como obter a sua densidade linear [1]. O segundo artigo utiliza a técnica de videoanálise no contexto de ondas estacionárias. Porém, essas circunstâncias exigem câmeras mais sofisticadas que filmem com 300 fps (frames per second), para fornecer resultados acurados da frequência e como consequência da velocidade de onda e da densidade linear do fio [2]. Os dois artigos seguintes abordam especificamente cordas de violão ou guitarra e estudam alguns parâmetros físicos e geométricos, usando também ondas estacionárias $[3,4]$.

Todos esses trabalhos têm em comum o uso de fios muito leves, cujos pesos são desprezíveis diante das tensões aplicadas. Diferentemente, o objetivo deste trabalho foi criar e analisar um experimento didático barato e eficiente para a obtenção da relação entre a velocidade e a tensão de um pulso e da densidade linear de uma corda, cuja massa não fosse desprezível. Nessa situação, a corda não fica em linha reta, mas pende devido ao seu próprio peso formando uma curva bem conhecida, dentro e fora da matemática, chamada catenária.

A catenária (do latim, catena significa corrente) tem uma história instigante. Em 1638, Galileu propôs que uma corrente suspensa pelas extremidades, sob a ação da força da gravidade, teria a forma de uma parábola. Contudo, Christiaan Huygens mostrou, sem provar, que essa curva não poderia ser a parábola. Em 1669, Joachim Jungius demonstrou que a catenária não era a parábola. Em 1690, Jakob Bernoulli lançou como desafio aos matemáticos determinar a equação da catenária. No ano seguinte, Christiaan Huygens, Gottfried Leibniz e Johann Bernoulli, usando técnicas distintas resolveram definitivamente o problema da catenária [5]. Um exemplo da curva catenária na engenharia é o de pontes suspensas simples, feitas de cordas e tábuas. Na arquitetura, é muito empregada a catenária invertida, como por exemplo, no arco de sustentação da estação de trens Keleti, em Budapeste e nos inúmeros arcos que apoiam o telhado da casa Milà projetada por Antoni Gaudí, em Barcelona [6].

Para a realização do experimento, foi escolhida uma corrente feita com anéis rígidos de material plástico, por ser de um material menos denso que o metal, de fácil transporte e acesso. A corrente foi pendurada, em cada extremidade, em dois dinamômetros comuns usados em laboratórios didáticos. O pulso foi gerado aplicando-se com uma régua de madeira uma pancada rápida, numa das extremidades da corrente. A propagação do pulso foi filmada com uma câmera simples de smartphone fixa em um tripé. Os vídeos foram analisados usando o software livre Tracker [7-9].

Para analisar os resultados do experimento foram criados dois modelos teóricos distintos. O primeiro é o mais simples e consiste em supor que a curvatura da corrente de plástico causada pelo seu próprio peso é desprezível. Logo, a corrente pode ser tratada como uma corda elástica horizontal presa nas extremidades. Pela sua simplicidade, esse modelo é adequado para turmas de ensino médio, visto que a ênfase é estabelecer relações funcionais entre grandezas, especificamente a tensão e a velocidade. O segundo modelo se aproxima mais da realidade, pois considera a curvatura da corda causada pelo seu peso, o que modifica as medidas de distância e de tensão. Esse modelo é mais refinado, preciso e acurado, com enfoque para turmas de nível superior, tanto pela modelagem teórica quanto pelo procedimento experimental, que são um pouco mais complexos.

Uma questão pertinente é se os resultados experimentais são consistentes com a modelagem matemática da catenária. A resposta dessa questão não é uma mera curiosidade, pois fornece um modo de executar a experiência, que combina a precisão e acurácia do segundo modelo, dispensando o uso do dinamômetro. De fato, esse procedimento matemático permite eliminar simultaneamente duas medidas necessárias nos modelos anteriores e que são as maiores fontes de incertezas: a tensão e o ângulo com referência ao plano horizontal. Além disso, se os valores obtidos pela modelagem da catenária forem compatíveis, dentro da margem de incerteza, com os valores medidos de tensão pelos dinamômetros, teremos uma verificação experimental de que a curva produzida no experimento é realmente uma catenária. 


\section{Modelo teórico}

A Fig. 1a exibe o diagrama das forças que atuam sobre um elemento genérico de massa $d m$, em uma corda de massa $m$ e comprimento $L$, em repouso, presa em suas extremidades. A corda é parametrizada por $s$, que representa a distância de um ponto qualquer da corda até a origem arbitrária. O ângulo $\theta(s)$ é formado pelo vetor da força de tensão variável $\boldsymbol{T}(s)$, suposta sempre tangente à corda em cada ponto, com o eixo $x$. Observando o diagrama de corpo livre (Fig. 1b) constata-se que a componente horizontal $\boldsymbol{T}_{x}$ é constante. Porém, a componente vertical $\boldsymbol{T}_{y}(s)$ varia, aumentando com o ângulo, e o somatório das forças de tensão e peso no eixo $y$ também devem ser nulo.

A equação cartesiana geral da catenária é [10]:

$$
y(x)=\frac{T_{x}}{\mu g} \cosh \left(\frac{\mu g}{T_{x}} x+C_{1}\right)+C_{2}
$$

sendo,

$$
\begin{gathered}
\mu=\frac{m}{L}, \\
T_{x}=T(s) \cos (\theta(s)),
\end{gathered}
$$

$g$ é a aceleração da gravidade e $C_{1}$ e $C_{2}$ são constantes que dependem das condições de contorno.

A equação cartesiana padrão de uma onda unidimensional em um meio não dispersivo é:

$$
\frac{\partial^{2} y}{\partial x^{2}}=\frac{1}{v^{2}} \frac{\partial^{2} y}{\partial t^{2}}
$$

sendo,

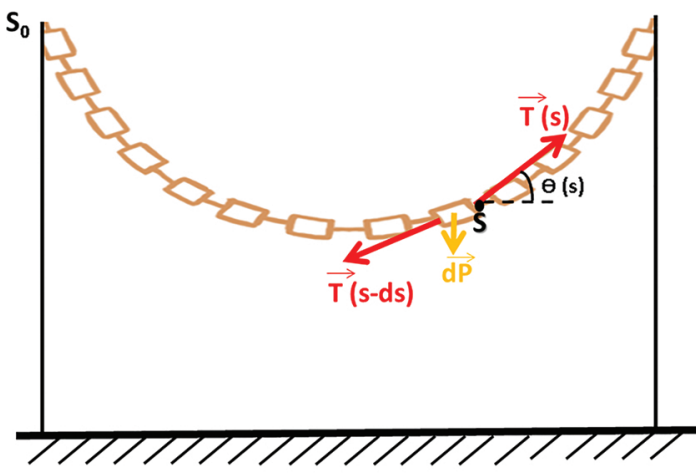

(1a)

$$
v^{2}=\frac{T}{\mu}
$$

A Eq. (4) só é válida para pequenas oscilações ortogonais à direção de propagação da onda e supondo que a força peso da própria corda seja desprezível em comparação com a tensão a que ela está submetida.

Para descrever pequenas oscilações em uma catenária é necessário levar em conta o peso da corda, gerando um sistema de equações não lineares acopladas, bem mais complicadas do que a equação de onda padrão. Para resolver esse sistema, pode-se considerar que o pulso foi produzido a partir da catenária estática e usar a suposição de que pequenas oscilações podem ser aproximadas por uma sucessão de configurações estáticas, pois as extremidades têm um deslocamento muito pequeno. Sob essas condições, é possível obter soluções numéricas, bem como a seguinte expressão para a velocidade de propagação da onda numa catenária [10]

$$
v^{2}(s)=\frac{T_{x}}{\mu} \sqrt{1+\frac{(\mu g s)^{2}}{T_{x}^{2}}} .
$$

O parâmetro $s$ foi assumido como sendo nulo no meio da corda (chamada de parametrização afim).

Alguns aspectos interessantes emergem da análise dessa equação. O primeiro é o valor da tensão, que na Eq. (5) é a tensão total, mas na Eq. (6) é somente a componente $x$ da tensão. A razão dessa discrepância é que a Eq. (5) supõe uma modelo unidimensional, isto é uma aproximação onde a corda é totalmente horizontal. Nesse caso, a componente $x$ da tensão é equivalente à própria tensão, sendo substituída pela última. Contudo, esse procedimento gera uma confusão, ao não deixar claro que a grandeza relevante

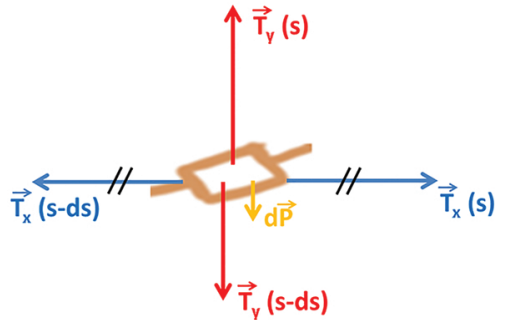

(1b)

Figura 1: A figura 1a mostra o diagrama das três forças que atuam em um elemento genérico de massa dm, em uma corrente de massa $\mathrm{m}$ e comprimento $\mathrm{L}$, em repouso, presa em suas extremidades. A figura $1 \mathrm{~b}$ mostra a força peso e as componentes horizontal e vertical das forças de tensão que atuam em um elemento de massa da corrente. 
na velocidade de propagação de qualquer onda numa corda é a apenas a componente da tensão paralela à direção de propagação.

O segundo aspecto é que, diferentemente da Eq. (5) que presume que a tensão e a velocidade de propagação do pulso são constantes, a Eq. (6) revela que uma pequena perturbação na catenária se propaga com uma velocidade variável, que é máxima nas extremidades e mínima no centro da corrente. Por fim, o último aspecto é a parametrização da catenária que não corresponde ao eixo $x$. Isso acarreta que a velocidade de propagação do pulso deve ser calculada usando distâncias medidas sobre a curva.

No caso de cordas submetidas a tensões bem superiores ao seu próprio peso, o termo $\mu g s / T_{x}=$ $\left(P / T_{x}\right)(s / L)<<1$ já que sempre $s / L<0,5$. Logo, a Eq. (6) pode ser expandida em série de Taylor e o valor da velocidade de uma onda na catenária até segunda ordem, torna-se:

$$
v^{2}(s)=\frac{T_{x}}{\mu}\left[1+\frac{\mu g s}{2 T_{x}}-\frac{(\mu \mathrm{gs})^{2}}{4 T_{x}^{2}}\right]
$$

Para estimar o efeito que os termos de primeira e segunda ordens provocam na medida de velocidade considere o seguinte exemplo com valores típicos dos parâmetros e grandezas envolvidos no experimento: o valor mínimo de tensão foi $\left.T_{x}=8,2 \sqrt{2}\right) \mathrm{N}$. O valor experimental da densidade linear, obtido dividindose a massa da corda pelo seu comprimento é $m=$ $0,1462(1) \mathrm{kg} / \mathrm{m}$. A aceleração da gravidade local é $g=9,78777 \mathrm{~m} / \mathrm{s}^{2}$ e o valor máximo do parâmetro é $s_{\max }=2,30(7) \mathrm{m}$. Usando esses valores na Eq. (7) os termos de primeira e segunda ordem podem ser desprezados. Nessa situação, a curvatura da catenária é desprezível, logo pode-se considerar $T_{x}=T$ e a Eq. (7) se reduz a Eq. (5). Essa aproximação acarreta um erro teórico percentual na velocidade que varia de $0 \%$, no meio da corda para aproximadamente $7,7 \%$, apenas nos pontos mais extremos. À medida que a tensão aumenta, a catenária se aproxima de uma linha horizontal, acarretando na diminuição do erro teórico.

\section{Procedimento experimental}

Uma corrente com anéis de material plástico de massa $m=0,77267(1) \mathrm{kg}$ e comprimento $L=5,285(2)$ $m$ foi escolhida por ser menos densa do que uma corda, mais simples de conectar aos dinamômetros e, principalmente, mais fácil de visualizar a frente de onda na análise quadro a quadro do vídeo. Cada extremidade da corrente foi conectada em dois dinamômetros metálicos em paralelo, previamente calibrados com massas de prova. A escala do dinamômetro estava em unidade de massa e o fundo de escala era 1,00 kg (equivalente a 9,7877 N, na gravidade local). Os dinamômetros foram presos a duas hastes metálicas verticais rígidas, encaixadas em pesadas bases de ferro e dispostos sobre superfícies horizontais (Figura 2).

Abaixo da corrente foi colocada uma régua padrão de 1,00 m com a finalidade de servir de escala para a realização da videoanálise. A régua foi colocada no mesmo plano da corrente para evitar o efeito de paralaxe que provoca um erro sistemático na construção da escala através do vídeo. Depois a

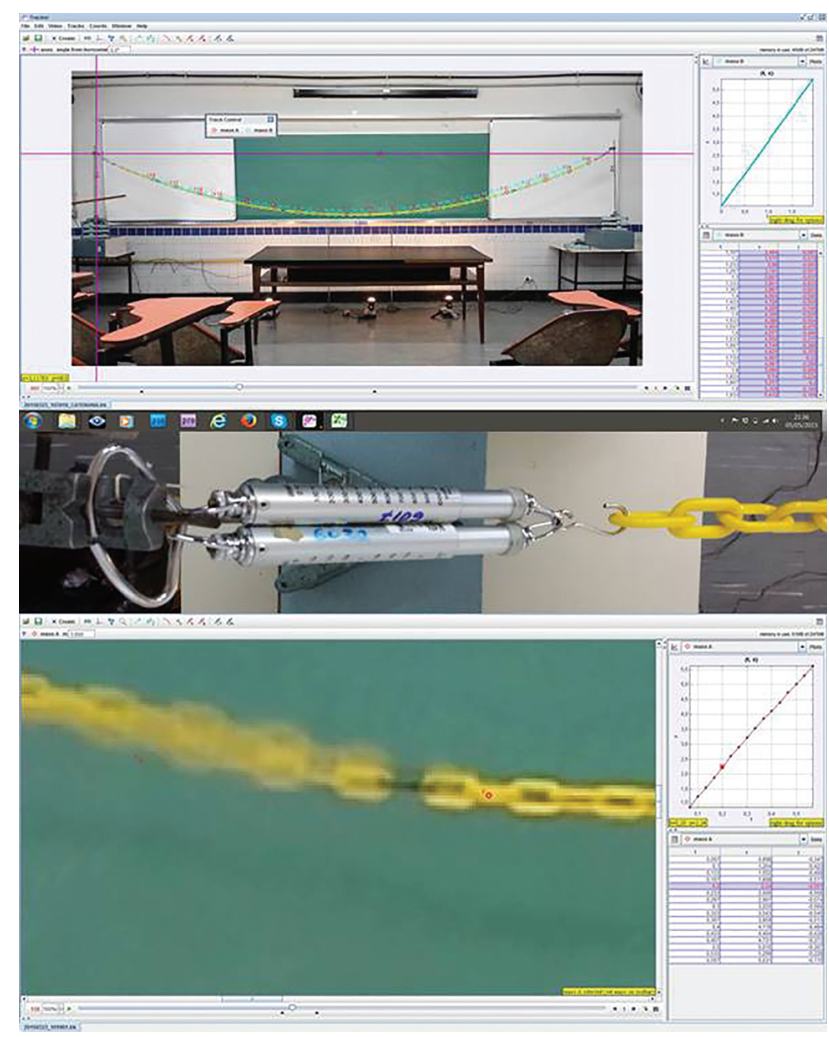

Figura 2: Captura de tela do Tracker, mostrando o aparato experimental, a corrente estática e a marcação dos pontos usados para fazer o ajuste teórico da catenária. Abaixo da corrente, no mesmo plano, está uma régua de comprimento $100 \mathrm{~cm}$ apoiada em dois suportes (foto superior). A corrente de plástico está presa nas suas extremidades por dois dinamômetros conectados em paralelo em hastes metálicas verticais formando um ângulo $\theta$ com a horizontal (foto central). Detalhe do movimento do pulso transversal mostrando a frente de onda representada pelo anel nítido a frente dos aneis borrados (foto inferior). 
corrente foi presa aos dinamômetros e ficou pendente no ar, submetida uma tensão baixa. Foram extraídos alguns anéis em ambas as extremidades até atingir a tensão mínima desejada, tomando o cuidado para que os valores marcados nos quatro dinamômetros fossem coincidentes entre si.

Uma questão técnica importante é sobre a utilização dos dinamômetros. Esses instrumentos foram projetados para funcionar em posição vertical. Consequentemente as suas escalas não são apropriadas para medidas com ângulos de inclinação próximos à direção horizontal. Essas medidas apresentam um erro sistemático causado por uma elongação deficiente da mola e fornecem resultados sempre abaixo dos valores reais. Para corrigir esse problema foi necessário fazer uma nova calibração do dinamômetro.

No nosso experimento, os dinamômetros variam de um ângulo de 25 graus para a menor tensão até 12 graus para a maior tensão, em relação à direção horizontal. Utilizando um conjunto de massas de prova, os dinamômetros foram submetidos às mesmas condições do experimento, de tensão e ângulo. Todos os resultados revelaram um erro sistemático negativo de, aproximadamente, $0,4 \mathrm{~N}$, que foi incorporado às medidas.

Além disso, quando os dinamômetros são usados fora das suas especificações, a mola interna arrasta no interior do tubo produzindo uma força de atrito estático que interfere na medida. Por exemplo, com os dinamômetros inclinados em relação ao eixo horizontal, a mesma massa de prova, não corresponde a um valor único na escala do dinamômetro, mas pode ser equilibrada dentro de uma faixa de valores, que varia com o ângulo. Esse intervalo deve ser considerado como a incerteza efetiva do instrumento, ao invés da incerteza da escala do aparelho. Convertendo-se as incertezas para unidade de força, essa faixa de imprecisão varia de acordo com a tensão e o ângulo, entre $0,3 \mathrm{~N}$ (menor tensão e maior ângulo) e 0,8 N (maior tensão e menor ângulo).

Foram escolhidos seis valores diferentes de tensão, marcados em todos os dinamômetros em unidades de massa, entre 920 gramas e 1.880 gramas (equivalente ao intervalo de $9,0 \mathrm{~N}$ e $18,4 \mathrm{~N}$ ). O critério de escolha da faixa de valores da tensão foi a praticidade. Para valores inferiores, a corrente ficava muito frouxa e o valor máximo de fundo de escala do conjunto (2.000 gramas) foi evitado, por precaução. As diferentes tensões foram ajustadas afastando-se uma base de ferro da outra, o que acarreta na dimi- nuição do ângulo do dinamômetro com a horizontal e o aumento da tensão.

Uma medida bastante sensível é o ângulo inicial que a corrente faz com a direção horizontal nas suas extremidades. Através desse ângulo, são obtidos os valores das projeções ortogonais da tensão. A componente $x$ da tensão está relacionada com a velocidade de propagação da onda e a componente $y$ deve equilibrar a metade do peso. O melhor procedimento para a medida desses ângulos é utilizar o sistema de coordenadas disponível no programa Tracker e girá-lo até que um de seus eixos esteja paralelo ao dinamômetro. A incerteza dessa medida foi estimada em apenas 1 grau. Dificilmente essa incerteza seria tão pequena caso se optasse pelo uso de um transferidor.

A filmagem foi realizada com um smartphone Samsung Galaxy S4 modelo GT-I9515 configurado com uma taxa de aquisição 30 quadros por segundo, full HD e resolução de 1920x1080 pixels. O aparelho foi fixado em um tripé e posicionado com vista frontal ao plano da catenária, a uma distância de aproximadamente 5 metros. Os vídeos da propagação dos pulsos foram transferidos para o computador e analisados utilizando o software livre Tracker.

Para cada valor de tensão, foi aplicada na parte inferior da extremidade esquerda da corda, uma pancada seca e rápida, com a face mais estreita de uma régua de madeira, transversal à corrente. Os pulsos gerados tinham baixa amplitude quando comparados ao comprimento da corrente, mas podiam ser facilmente identificados. Apenas o trecho do movimento antes da reflexão na outra extremidade foi utilizado na videoanálise.

Para medir a posição do pulso se propagando e minimizar o efeito de perda de nitidez do zoom do vídeo, foi considerado como frente de onda, o anel da corrente que estava nítido, imediatamente adiante do anel que estava borrado devido ao deslocamento vertical, provocado pela passagem do pulso. A figura 2 ilustra a captura de tela do Tracker, mostrando os pontos que marcam a posição do pulso sobre a corrente. A incerteza da posição foi obtida por estimativa, de forma conservadora, como sendo de um anel da corrente, o que equivale a $\pm 5 \mathrm{~cm}$.

\section{Análise de resultados}

Do ponto de vista didático, foram elaborados dois modelos teóricos diferentes para analisar os resul- 
tados do experimento. Em ambos os modelos, as medidas das posições e tempo do movimento do pulso geradas pelo Tracker foram tabuladas e tratadas no programa de gráficos Origin. Como previsto teoricamente, a variação da velocidade do pulso ao longo da catenária foi muito pequena e os pontos experimentais foram ajustados através de uma tendência linear.

As velocidades de propagação do pulso para diferentes tensões foram obtidas extraindo-se o coeficiente angular dos ajustes e as suas incertezas foram obtidas pelo método dos mínimos quadrados. A Tabela 1 resume as medidas de velocidade usando os pontos experimentais sobre a catenária $(v)$ e as medidas de velocidade utilizando as posições dos pontos experimentais sobre o eixo $x\left(v_{x}\right)$, bem como as incertezas associadas. Quanto maior a tensão, mais esticada fica a corrente e, portanto os valores de $v$ e $v_{x}$ se aproximam até coincidirem, dentro da margem de incerteza.

A velocidade total $v$ foi calculada considerando os módulos dos deslocamentos horizontal e vertical da frente de onda, ao longo da corrente. A velocidade $v_{x}$ foi obtida apenas considerando a posição da frente de onda no eixo $x$, como se a corrente tivesse totalmente na horizontal. Essa última situação é a aproximação de massa desprezível, empregada nos livros didáticos de ensino médio e superior.

\subsection{Primeiro modelo}

Modelo mais simples que consiste em supor que a tensão na corrente será constante ao longo de todos os seus anéis e igual à fornecida por um dinamômetro preso a uma de suas extremidades. Logo, a corrente pode ser tratada como uma corda horizontal presa nas extremidades. A velocidade de propagação do pulso é considerada constante, por definição. A tensão é suposta atuar na direção horizontal, em

Tabela 1: Valores das velocidades de propagação do pulso usando os pontos experimentais sobre a catenária (v) e as posições dos pontos sobre o eixo $\mathrm{x}\left(\mathrm{v}_{x}\right)$ e as incertezas associadas.

\begin{tabular}{lllll}
\hline$T(\mathrm{~N})$ & $v^{2}(\mathrm{~m} / \mathrm{s})^{2}$ & $\delta v^{2}(\mathrm{~m} / \mathrm{s})^{2}$ & $v_{x}^{2}(\mathrm{~m} / \mathrm{s})^{2}$ & $\delta v_{x}^{2}(\mathrm{~m} / \mathrm{s})^{2}$ \\
\hline 9,0 & 60,3 & 0,8 & 58 & 0,8 \\
11,0 & 70,9 & 0,7 & 69 & 0,7 \\
12,9 & 89 & 1 & 88 & 1 \\
14,9 & 107 & 2 & 107 & 2 \\
16,8 & 121 & 2 & 121 & 2 \\
18,4 & 131 & 2 & 130 & 2 \\
\hline
\end{tabular}

todos os pontos. A parametrização das distâncias na corda é simplesmente a projeção horizontal da sua posição (o parâmetro é a própria coordenada $x$ ). Esse modelo é útil como primeira aproximação e também satisfatório para turmas cuja ênfase é estabelecer correlações entre as grandezas, sem a preocupação com a acurácia.

A Tabela 2 contém os resultados das medidas de ângulo dos dinamômetros com a direção horizontal, tensão total e suas componentes e respectivas incertezas.

A descrição das incertezas de ângulo e tensão está no procedimento experimental. Por outro lado, as incertezas das componentes cartesianas da tensão, foram obtidas por propagação de incertezas. Percebese que os módulos de $T_{y}$ se mantêm constantes, dentro da margem de incerteza. Isso ocorre porque estamos calculando a componente vertical da tensão nas extremidades, cujo valor deve ser fixo e igual à metade do peso da corrente.

A figura 3 mostra o gráfico da tensão total medida pelos dinamômetros versus o quadrado da velocidade de propagação do pulso, considerando as posições sobre o eixo $x$. O gráfico contém os dados de tensão da Tabela 2 e de $v_{x}$ da Tabela 1.

O coeficiente angular do gráfico na figura 3 fornece a densidade linear da corrente $\mu_{\text {exp }}=0,124(5) \mathrm{kg} / \mathrm{m}$. Consideramos como valor de referência aquele calculado diretamente pela razão entre a massa e o comprimento da corrente, que vale $\mu_{\text {direto }}=0,1462(1)$ $\mathrm{kg} / \mathrm{m}$. A comparação entre eles fornece um erro relativo percentual de $-15,2 \%$.

Podemos concluir que o primeiro modelo tem êxito em mostrar a relação linear entre tensão e quadrado da velocidade. Contudo, o resultado experimental da densidade linear da corrente é pouco acurado. $\mathrm{O}$ custo benefício do modelo é bom, porque as medidas são simples, os resultados qualitativos são confiáveis e os quantitativos são aceitáveis. Esse modelo é ideal para turmas de ensino médio.

\subsection{Segundo modelo}

Este modelo é mais realista, pois leva em conta a curvatura da corda causada pelo seu peso. Essa abordagem incorpora dois aspectos relevantes no modelo teórico. O primeiro é que a tensão é variável e tangente à corda em cada ponto e a sua componente vertical deve equilibrar o peso de um elemento de massa (anel) da corrente. Consequentemente, apenas a componente horizontal constante da tensão 
Tabela 2: Valores dos ângulos dos dinamômetros com a direção horizontal, tensões totais e as suas componentes e as incertezas associadas.

\begin{tabular}{llllllll}
\hline Angulo $(\theta)$ & $\delta \theta$ & Tensão $T(\mathrm{~N})$ & $\delta T(\mathrm{~N})$ & $T_{x}(\mathrm{~N})$ & $\delta T_{x}(\mathrm{~N})$ & $T_{y}(\mathrm{~N})$ & $\delta T_{y}(\mathrm{~N})$ \\
\hline $25^{0}$ & $1^{0}$ & 9,0 & 0,3 & 8,2 & 0,2 & 3,8 & 0,1 \\
$22^{0}$ & $1^{0}$ & 11,0 & 0,4 & 10,2 & 0,4 & 4,1 & 0,2 \\
$17^{0}$ & $1^{0}$ & 12,9 & 0,5 & 12,4 & 0,5 & 3,8 & 0,2 \\
$16^{0}$ & $1^{0}$ & 14,9 & 0,6 & 14,3 & 0,5 & 4,1 & 0,2 \\
$14^{0}$ & $1^{0}$ & 16,8 & 0,8 & 16,3 & 0,8 & 4,1 & 0,3 \\
$12^{0}$ & $1^{0}$ & 18,4 & 0,8 & 18,0 & 0,8 & 3,8 & 0,3 \\
\hline
\end{tabular}

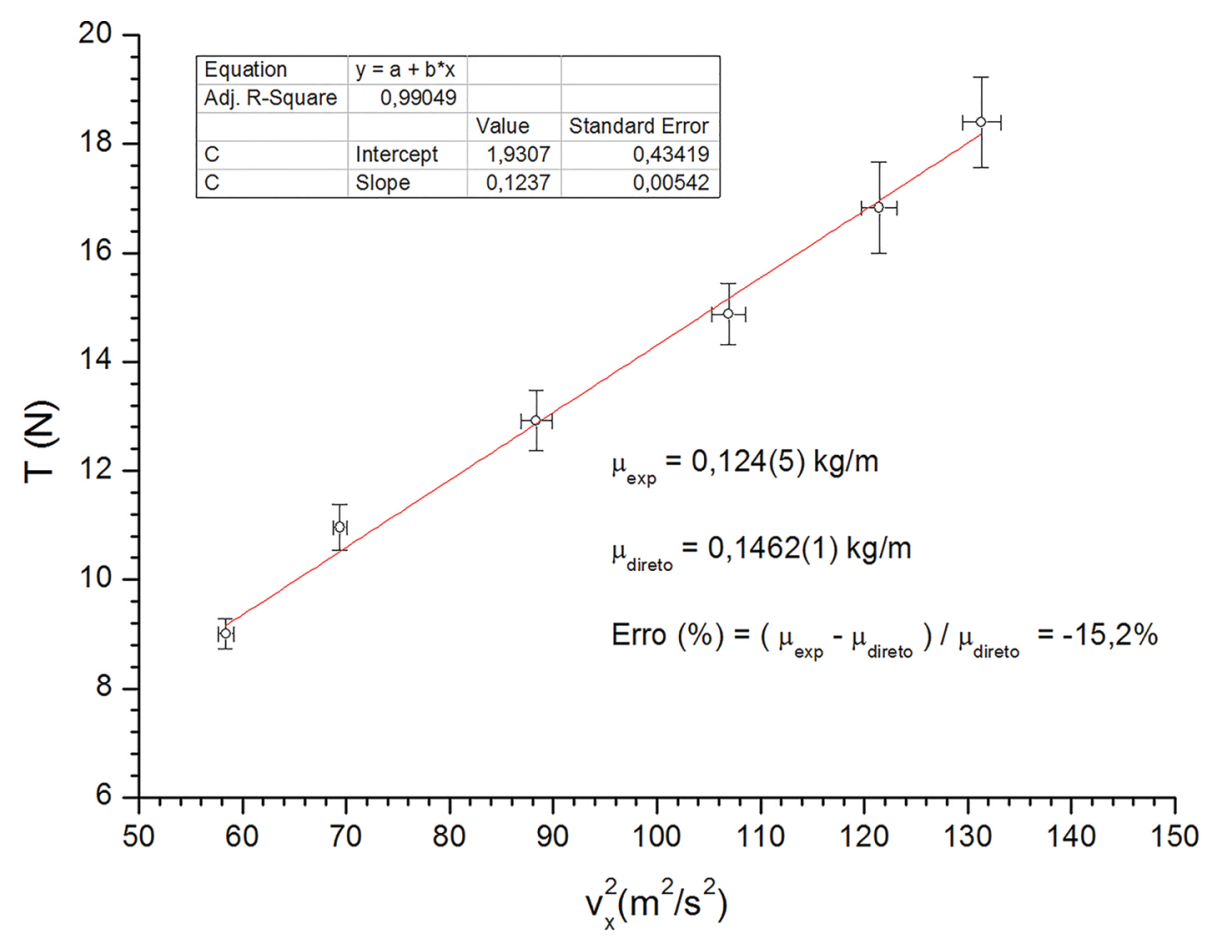

Figura 3: Gráfico da tensão total medida pelos dinamômetros em função do quadrado da velocidade do pulso no eixo x, isto é, considerando a corrente horizontal.

deve ser considerada no cálculo da velocidade de propagação do pulso. O segundo é que as distâncias na corda são calculadas usando um parâmetro ao longo da catenária (parâmetro $s$ ), tornando o tratamento de dados um pouco mais complicado.

Na prática, as distâncias ao longo da catenária são calculadas pelo teorema de Pitágoras aplicado às componentes horizontal e vertical de um deslocamento infinitesimal. Depois, cada deslocamento resultante é adicionado ao valor do deslocamento anterior. Todo o procedimento é feito automaticamente por um programa de planilha eletrônica, a partir dos dados de posição importados do Tracker.

A figura 4 exibe o gráfico da componente horizontal da tensão total medida pelos dinamômetros versus o quadrado da velocidade de propagação do pulso, considerando apenas as posições dos pontos experimentais sobre a catenária. O gráfico foi gerado com os dados de $T_{x}$ da Tabela 2 e de $v$ da Tabela 1 .

A densidade linear da corrente no segundo modelo é $\mu_{\text {exp }}=0,135 \sqrt{6} \mathrm{~kg} / \mathrm{m}$ e o seu erro relativo percentual ao valor de referência é de -7,7\%. Comparando com o primeiro modelo, houve uma redução do erro relativo percentual quase pela metade. Isso demonstra como é relevante o efeito da massa da corrente nos resultados provenientes da modelagem teórica. Esse modelo é mais apropriado para turmas de ensino superior.

Existe uma alternativa para simplificar o aparato experimental que dispensa o uso dos dinamômetros. Nesse caso, a componente horizontal da tensão é obtida indiretamente através da componente vertical na extremidade, que é igual à metade do peso total da corrente. Na verdade, a componente vertical varia 


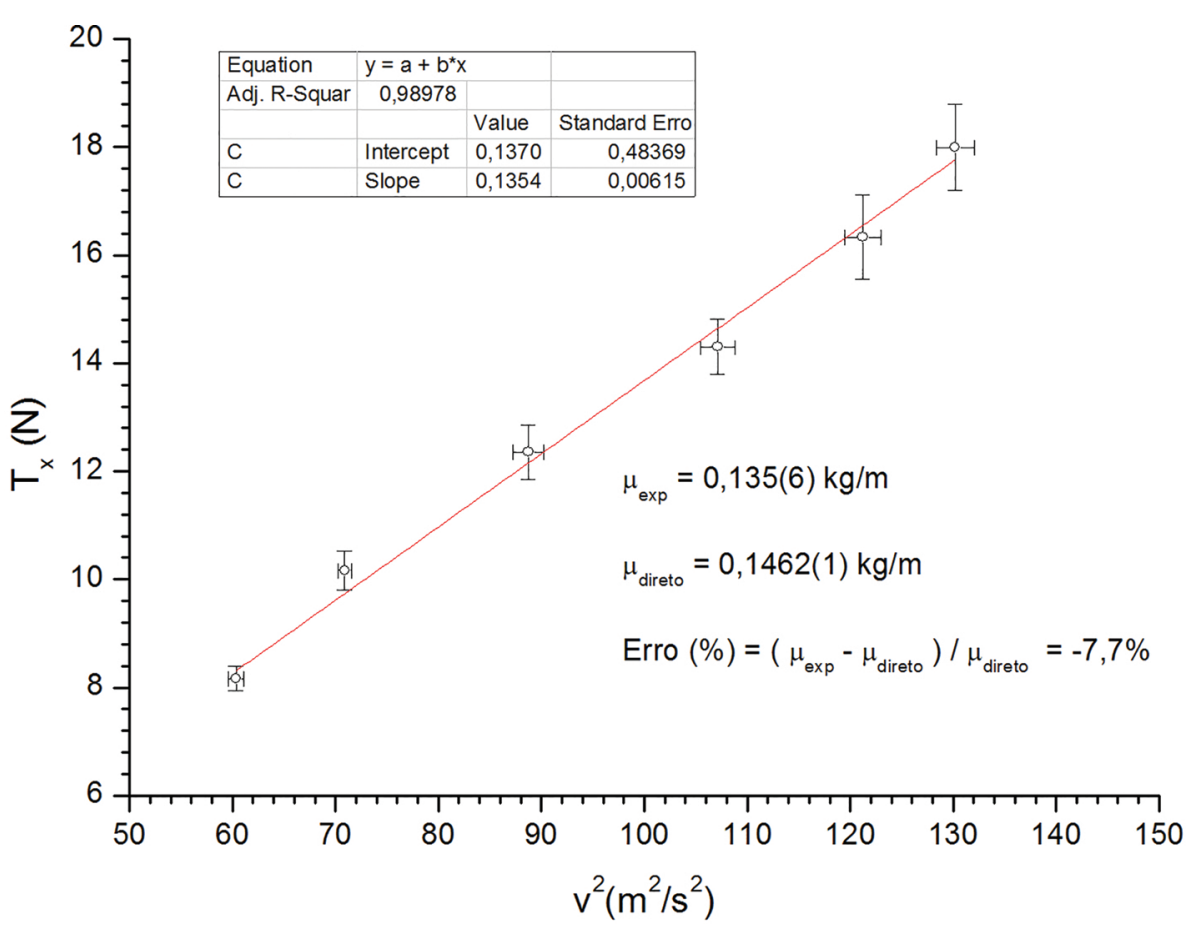

Figura 4: Gráfico da componente horizontal da tensão total medida pelos dinamômetros em função do quadrado da velocidade total, isto é, considerando que a corrente faz uma catenária.

em cada ponto da corda, portanto, essa aproximação vai acarretar numa perda de acurácia semelhante ao primeiro modelo. Uma desvantagem adicional é que as componentes da tensão se relacionam através da função tangente, cuja derivada é muito acentuada. Então, mesmo a pequena incerteza de 1 grau na medida do ângulo vai acarretar numa incerteza elevada da componente horizontal da tensão. $\mathrm{Na}$ soma dos efeitos, esse é a pior escolha, tanto em precisão quanto em acurácia. A sua única virtude é simplificar consideravelmente a experiência.

O peso total da corrente é $P=7,5627(1) \mathrm{N}$ ), logo a componente vertical da tensão total nas suas extremidades é $\left.T_{y}=3,78135(5) \mathrm{N}\right)$. Considerando os ângulos descritos na Tabela 2, é possível obter os valores de $T_{x}$, a partir da seguinte equação:

$$
T_{x}=\frac{T_{y}}{t g \theta}
$$

A figura 5 exibe o gráfico da componente horizontal da tensão obtida pela Eq. (8) versus o quadrado da velocidade de propagação do pulso, considerando apenas as posições dos pontos experimentais sobre a catenária.

A densidade linear da corrente obtida sem o uso dos dinamômetros é $\mu_{\text {exp }}=0,123(9) \mathrm{kg} / \mathrm{m}$ e o seu erro relativo percentual ao valor de referência é de
-15,9\%. Comparado ao primeiro modelo, a acurácia é equivalente, mas a incerteza é maior. Sem dúvida, ele é indicado apenas quando não se dispõe de dinamômetros.

\section{Relação entre as medidas experimen- tais e equação da catenária}

A forma cartesiana da catenária, Eq. (1), contém três parâmetros físicos e duas constantes fixadas pelas condições de contorno. Então, através de um ajuste teórico das medidas de posição da corda obtidas pelo Tracker pode-se extrair matematicamente a componente horizontal da tração, sem a necessidade de medir a força diretamente. Esse refinamento teórico possibilita a eliminação das medidas de tensão e ângulo, que são as causas das maiores incertezas do experimento.

A configuração da catenária estática foi mapeada no Tracker, que gerou automaticamente uma tabela de pares ordenados de posição nos eixos $x$ e $y$. Os dados foram exportados para o programa Origin, onde foi feito um gráfico impondo o ajuste à Eq. (1). Os valores da aceleração da gravidade e da densidade linear foram inseridos para que as outras constantes sejam calculadas, tais como a componente horizontal da tensão. Esse procedimento foi repetido para os 


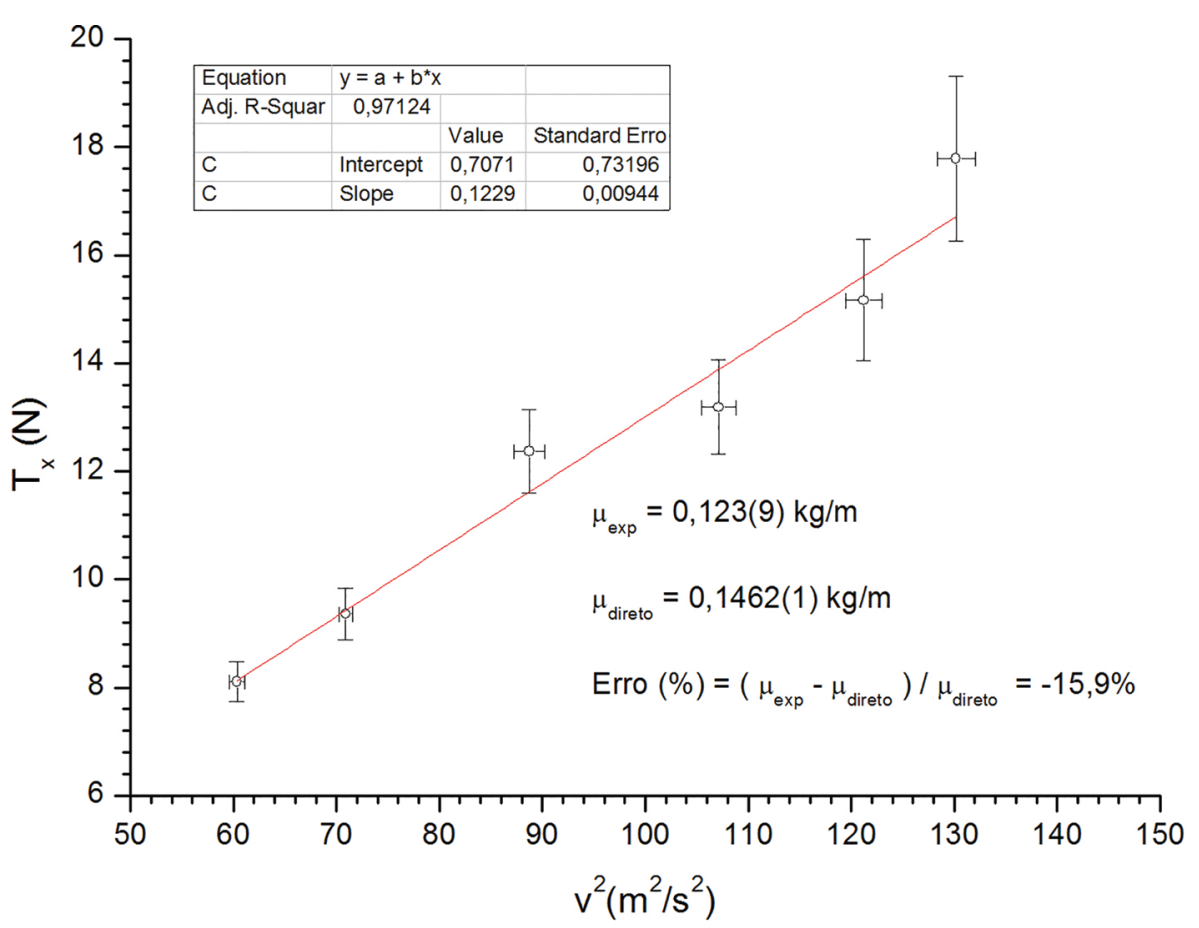

Figura 5: Gráfico da componente horizontal da tensão total, obtida indiretamente por medida do peso total da corrente, em função do quadrado da velocidade total, isto é, considerando que a corrente forma uma catenária.

seis valores de tensão. Embora seja aparentemente trabalhoso, esse método é mais rápido e simples do que calibrar dinamômetros, fazer medidas de tensão e ângulo e propagar incertezas.

Na Tabela 3 estão os resultados da componente $x$ da tensão obtidos pelo ajuste matemático da catenária, bem como de suas respectivas incertezas. A incerteza da componente horizontal da tensão está na segunda casa decimal, sendo uma ordem de grandeza melhor do que os modelos anteriores. Como comparação, também estão arrolados os valores da componente $x$ da tensão obtidos através das medidas de tensão pelos dinamômetros e de ângulo. Existe uma superposição dos valores entre os dois modelos, dentro da margem de incerteza. Esse fato pode ser considerado uma confirmação experimental de que o ajuste matemático foi bem sucedido, isto é, a curva feita pela corrente sob a ação do seu próprio peso é realmente uma catenária.

A figura 6 exibe o gráfico da componente horizontal da tensão total obtida pelo ajuste matemático da catenária versus o quadrado da velocidade de propagação do pulso, considerando apenas as posições dos pontos experimentais sobre a catenária. O gráfico foi gerado com os dados de $T_{x}$ da Tabela 3 e de $v$ da Tabela 1.

Nesse caso, a densidade linear da corrente é $\mu_{\text {exp }}$
$=0,137 \sqrt{6} \mathrm{~kg} / \mathrm{m}$ e o seu erro relativo percentual ao valor de referência é de $-6,3 \%$, a menor diferença, como era esperado. Uma dificuldade, que pode ser convertida em uma interessante discussão em sala de aula, é que esse ajuste exige o conhecimento de algumas propriedades da catenária, incluindo a sua equação cartesiana. Por esse motivo, indicamos esse procedimento apenas para turmas de graduação em física e engenharias.

\section{Considerações finais}

Em geral, as atividades didáticas experimentais sobre pulsos e ondas em cordas estão baseadas na validade da hipótese de um fio horizontal, cujo peso é desprezível em relação às forças de tensão. Essa aproximação simplifica tanto a equação de onda, tornando-a linear, como a relação entre a tensão e a velocidade da onda. Entretanto, esse procedimento restringe severamente os experimentos e frequentemente exige o uso de sensores e/ou equipamentos mais caros para extrair dados confiáveis.

O diferencial deste trabalho foi sair do lugar comum e fazer uma experiência de baixo custo e fácil acesso, mas com resultados de boa qualidade sobre a propagação de um pulso em uma corrente plástica, que se deforma sob a ação do próprio peso, formando 
Tabela 3: Valores das componentes horizontais da tensão e as incertezas associadas, obtidas pelo ajuste matemático da catenária e pela medida de dinamômetro e ângulo.

\begin{tabular}{llll}
\hline$T_{x}(\mathrm{~N})$ Catenária & $\delta T_{x}(\mathrm{~N})$ Catenária & $T_{x}(\mathrm{~N})$ Dinamômetro & $\delta T_{x}(\mathrm{~N})$ Dinamômetro \\
\hline 8,09 & 0,01 & 8,2 & 0,2 \\
10,01 & 0,02 & 10,2 & 0,4 \\
12,23 & 0,03 & 12,4 & 0,5 \\
14,48 & 0,03 & 14,3 & 0,5 \\
16,54 & 0,04 & 16,3 & 0,8 \\
17,89 & 0,06 & 18,0 & 0,8 \\
\hline
\end{tabular}

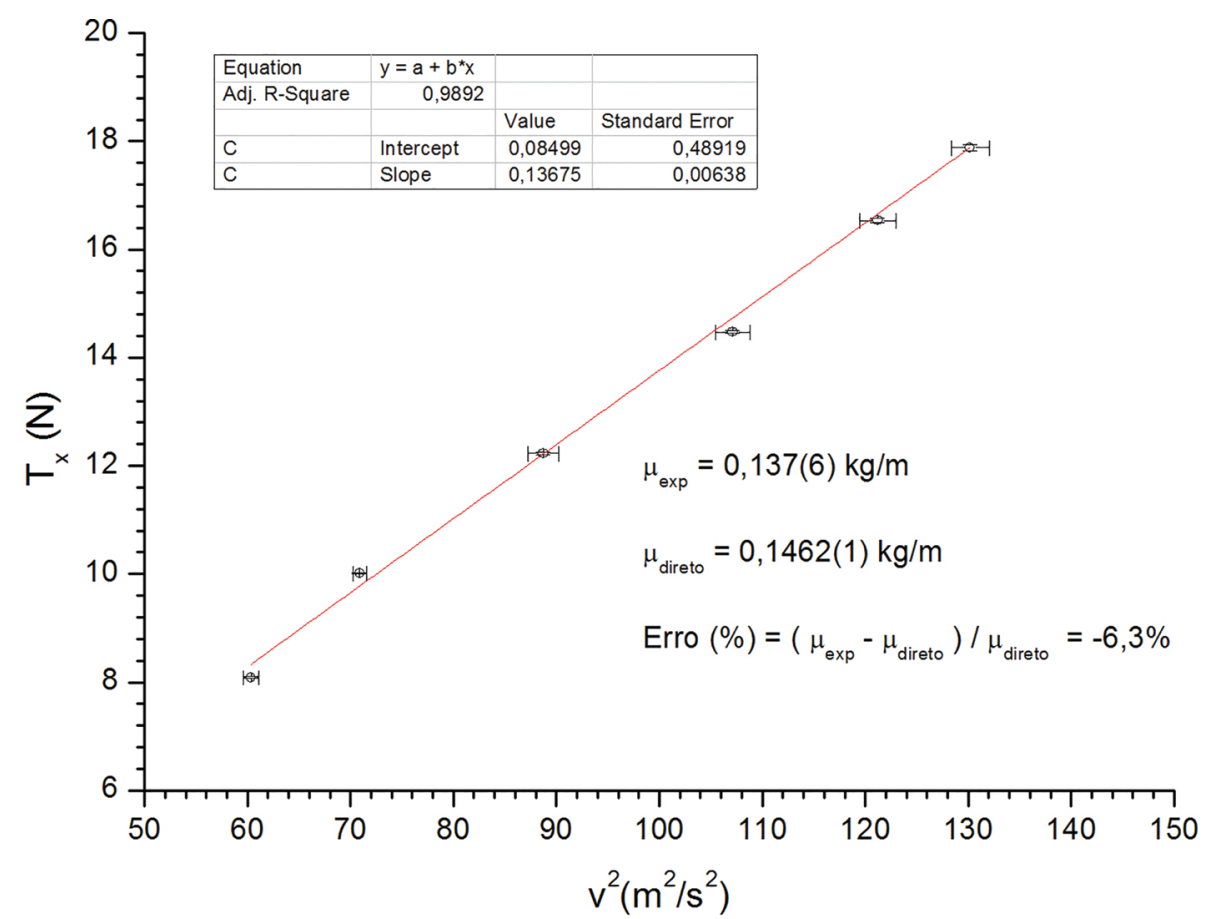

Figura 6: Gráfico da componente horizontal da tensão total, obtida indiretamente pelo ajuste matemático da equação da catenária em função do quadrado da velocidade total, isto é, considerando que a corrente forma uma catenária.

a conhecida forma geométrica da função catenária. De fato, usando uma câmera de filmagem comum de um smartphone e as ferramentas de um software gratuito de videoanálise, é possível realizar uma experiência sobre a velocidade de propagação de um pulso em uma catenária, bem como extrair a sua densidade linear.

Os dois modelos teóricos aplicados na interpretação dos resultados permitiram uma compreensão mais ampla e profunda do experimento. Além disso, o desenvolvimento de diferentes formas de modelar um mesmo fenômeno, com uma gradação de complexidade, proporciona abordagens ajustadas tanto para o nível médio quanto para diversos cursos de graduação. A análise dos resultados revelou uma variação da acurácia e precisão de acordo com os dois modelos matemáticos adotados. Isso possibilitou uma interessante visualização de que o trabalho ex- perimental não depende somente dos equipamentos empregados, mas é indissociavelmente entrelaçado com as respectivas teorias.

\section{Agradecimentos}

Os autores agradecem à Caroline Alves Cayres pela confecção da primeira figura.

\section{Referências}

[1] T. Odekirk and W.V. Slaton, The Physics Teacher 50, 244 (2012).

[2] M.A. Cavalcante, R. Peçanha e A.C. Teixeira, Revista Brasileira de Ensino de Física 35, 3502 (2013).

[3] F. Catelli e G.A. Mussato, Revista Brasileira de Ensino de Física 36, 1306 (2014).

[4] F. Catelli e G.A. Mussato, Revista Brasileira de Ensino de Física 36, 2304 (2014). 
[5] J.F. Bukowski, College Math Journal 39, 2 (2008).

[6] Informação pessoal fornecida por um dos autores que visitou os locais citados.

[7] http://www.cabrillo.edu/ dbrown/tracker/, acessado em 30/9/2015.

[8] D. Brown and A.J. Cox, The Physics Teacher 47, 145 (2009).

[9] A.G. Bezerra Júnior, J.A. Lenz, L.P. Oliveira e N. Saavedra, Manual para Usuários Iniciantes no Software Tracker (Universidade Tecnológica Federal do Paraná, Curitiba, 2011).

[10] D. Klerk, J. Murugan and J.P. Uzan, arXiv:1103. 0788 [physics.class-ph] (2011). 